\title{
Factors Affecting Genetic Consultation in Adolescent and Young Adult Patients With Sarcoma
}

\author{
Grace E. McKay, MPH${ }^{1}$; Anna L. Zakas, MS, MPH, CGC ${ }^{2}$; Fauzia Osman, MPH ${ }^{1}$; and Amanda Parkes, MD ${ }^{1}$
}

\begin{abstract}
Background: Given a link between sarcomas and hereditary cancer predisposition syndromes, including Li-Fraumeni syndrome, the consideration for genetic counseling is recommended for all adolescent and young adult (AYA) patients diagnosed with sarcoma. The aim of this study was to evaluate factors influencing genetic consultations in AYA patients with sarcoma at the University of Wisconsin (UW). Methods: A retrospective chart review was performed on AYA patients diagnosed with sarcoma between the ages of 15 and 39 years who were seen at least once between 2015 to 2019 at UW. Our chart review identified discussions regarding genetics, referrals to genetics, genetic consultations, and results of genetic testing. Variables hypothesized to affect patient referrals for genetic consultation were identified a priori. Descriptive statistical methods and a univariate analysis were used to identify patient characteristics associated with genetic counseling referral. Results: We identified 87 AYA patients with sarcoma. Only 19 (22\%) of these patients had documentation of a discussion about genetics, 15 (17\%) of whom were subsequently referred for genetic consultation. Of these 15 patients, 9 $(60 \%)$ were seen in consultation. All 9 patients seen by genetics underwent genetic testing, with 4 (44\%) of these patients having identified heritable cancer predisposition syndromes. Likelihood for genetics referral was linked most strongly to documented genetics discussion with an oncology provider $(P<.001)$. Conclusions: Despite the recommendation for consideration for genetic counseling in AYA patients with sarcoma, $<25 \%$ of such patients in our study had a documented discussion about genetics. Supporting this need, all referred patients met criteria for genetic testing, and $44 \%$ of tested patients were found to have a heritable cancer predisposition syndrome. These data support the initial conversation by a provider as critical to genetic referral and suggest the need for more specific national recommendations for the genetic evaluation of all AYA patients with sarcoma.
\end{abstract}

J Natl Compr Canc Netw, doi: 10.6004/jnccn.2021.7034 Published online October 19, 2021

${ }^{1}$ Section of Hematology/Oncology, Department of Medicine, and ${ }^{2}$ Section of Oncology Genetics, Department of Pediatrics, University of Wisconsin-Madison School of Medicine and Public Health, Madison, Wisconsin.

\section{Background}

The incidence of cancer in adolescents and young adults (AYAs) aged 15 to 39 years has been increasing steadily. ${ }^{1,2}$ Although a rare tumor type, sarcomas are seen with relative frequency in AYAs, accounting for approximately $14 \%$ of all AYA cancer diagnoses. ${ }^{3}$ The disproportionate frequency of sarcomas that affect AYAs has been suggested as support for a genetic basis of hereditable conditions in patients with sarcoma, which is also strengthened by the increased risk of second cancers seen in sarcoma survivors. ${ }^{4}$ Consistent with this, studies have shown a large burden of genetic risk in patients with sarcoma, ${ }^{5,6}$ including a 2016 study showing that $55 \%$ of 1,162 individuals with sarcoma had an excess of pathogenic germline variants. ${ }^{7}$

Given the known association of sarcomas with several genetic syndromes, including Li-Fraumeni syndrome (LFS), ${ }^{8,9}$ consideration for genetic counseling is recommended for all AYA patients diagnosed with sarcoma. ${ }^{10,11}$ Since 2015, the NCCN Clinical Practice Guidelines in Oncology (NCCN Guidelines) for Genetic/Familial HighRisk Assessment: Breast, Ovarian, and Pancreatic ${ }^{10}$ have recommended genetic consultation with possible genetic testing for patients with sarcoma meeting classic LFS criteria $^{12}$ or Chompret criteria. ${ }^{13,14}$ Beyond LFS-specific guidelines, the NCCN Guidelines for Soft Tissue Sarcoma review the increased incidence of sarcoma seen in patients with LFS, hereditary nonpolyposis colorectal cancer, neurofibromatosis type 1 (NF1), Carney-Stratakis syndrome, familial adenomatous polyposis (FAP), and hereditary retinoblastoma. ${ }^{15}$ These guidelines, however, provide no generalized referral strategies for patients with sarcomas and offer only syndrome-specific evaluation considerations. The NCCN Guidelines for AYA Oncology more broadly recommend that a thorough family history be taken, preferably by a genetic counselor ${ }^{11}$; however, ongoing work has shown that barriers remain to genetic consultation and appropriate cancer risk management. ${ }^{16-18}$

Given the high prevalence of hereditary cancer predisposition syndromes in AYA patients and patients with sarcoma and the critical importance of genetic consultation in these patients, we sought to understand referral patterns for genetic consultation and subsequent genetic testing in 
AYA patients with sarcoma at the University of Wisconsin (UW). We sought to evaluate factors influencing genetic consultations in these patients to seek quality improvement opportunities to increase the rate of genetic counseling referrals.

\section{Methods}

\section{Medical Record Retrieval}

AYA patients with cancer between the ages of 15 and 39 years were identified via electronic health record (EHR) query using age, any cancer diagnosis recorded in hospital or professional billing, and at least 1 visit in 2015 through 2019 at the UW Carbone Cancer Center (UWCCC) or American Family Children's Hospital (AFCH) with a medical or radiation oncologist. Subsequent physician-led chart review identified patients with sarcoma for study inclusion. Study data were collected via retrospective chart review and managed via a secure institutional database. The study was conducted in accordance with all relevant guidelines and procedures and was approved by the UW Institutional Review Board as a quality improvement project.

\section{Retrospective Chart Review}

A retrospective chart review was performed, identifying documentation of discussions regarding genetics, referrals to genetics, genetic consultations, and results of genetic testing. The EHR search function was used to identify discussions regarding genetics by any oncology provider, including physicians, advanced practice providers, and nurses in medical or radiation oncology, and documentation of a referral to genetics. Referrals were also identified by review of EHR orders. Genetic consultation visits were confirmed by review of appointments and consultation notes. Variables hypothesized to affect patient referrals for genetic consultation were identified a priori and included age at diagnosis, sex, race, ethnicity, zip code of patient's residential location categorized into metropolitan versus nonmetropolitan residential location using Rural-Urban Continuum Codes, ${ }^{19}$ insurance status, localized versus metastatic cancer at time of diagnosis, receipt of chemotherapy, and documentation of a genetics discussion with an oncology provider.

\section{Statistical Analysis}

Patient characteristics were recorded as percentages for categorical variables and as means and standard deviations for continuous variables. Categorical data were assessed using Fisher exact tests, and continuous variables were compared using one-way ANOVA. We used a univariate analysis to identify variables associated with an increased rate of referral for genetic counseling. All $P$ values $\leq .05$ were considered statistically significant. STATA, version 16 SE (StataCorp LLP) was used to conduct all statistical analyses.

\section{Results}

\section{Patient Baseline Characteristics}

We identified 87 AYA patients with sarcoma who were seen at least once in 2015 through 2019 at UWCCC or AFCH by a medical or radiation oncologist. Just more than half of the patients were male $(54 \% ; n=47)$, and most identified as White $(91 \% ; \mathrm{n}=79)$ and non-Hispanic $(97 \% ; n=84)$. Most patients had health insurance $(91 \%$; $\mathrm{n}=79)$, with only $19 \%(\mathrm{n}=15)$ of these having Medicaid or Medicare as their primary health insurance (Table 1).

Table 2 details histologic subtypes seen in the 87 patients, with localized disease noted at the time of diagnosis in most patients $(85 \%$; $n=74)$. More than two-thirds of patients $(69 \% ; n=60)$ underwent chemotherapy as part of their treatment regimen, whereas fewer than half of patients $(45 \%$; $n=39)$ underwent radiation therapy. Most of these treatments were administered by the adult oncology team at UWCCC $(84 \% ; n=73)$, with only 14 patients $(16 \%)$ treated at AFCH by the pediatric oncology team.

\section{Primary Outcomes}

Of the 87 AYA patients with sarcoma identified in our study, only $22 \%(n=19)$ had documentation of a discussion about genetics with an oncology provider. There was no statistically significant difference in genetic discussion documentation by adult providers $(21 \%$; $15 / 73)$ versus pediatric providers $(29 \% ; 4 / 14)$, nor was there a statistically significant difference in medical versus radiation oncology providers (documentation in 22\% [16/74] and 23\% [3/13], respectively). Of the 19 patients with documented genetics discussions, 15 were subsequently referred for genetic consultation $(79 \%)$, which represented only $17 \%$ of the total study population (15/87). No patients lacking documentation of a genetics discussion with an oncology provider were referred for a genetics consultation. Of the 4 patients with a documented discussion about genetics but lack of genetics referral, only 1 (25\%) with spindle cell sarcoma had documentation of not being referred for genetic consultation because the medical provider believed that the patient's personal and family history of cancer did not meet requirements for genetic counseling referral. Of the remaining 3 patients, 2 had a lack of follow-up by the referring provider and $1 \mathrm{had}$ an unclear reason for lack of genetics referral.

Of the 15 patients referred to genetics, $60 \%$ were seen in consultation ( $n=9$ ) (Figure 1). Reasons for referral without subsequent consultation were lack of follow-up by patient (4 patients did not return paperwork required to schedule genetic consultation appointment), lack of 


\begin{tabular}{|c|c|c|c|c|}
\hline Characteristic & $\begin{array}{l}\text { Total } \\
\text { n (\%) }\end{array}$ & $\begin{array}{l}\text { Referred to Genetics } \\
\text { n (\%) }\end{array}$ & $\begin{array}{l}\text { Not Referred to Genetics } \\
\text { n (\%) }\end{array}$ & $P$ Value \\
\hline Total, $\mathrm{n}$ & 87 & 15 & 72 & \\
\hline Age at diagnosis, mean [SD] & $26.7[7.3]$ & $27[6.8]$ & $26.6[7.4]$ & .86 \\
\hline Sex & & & & .78 \\
\hline Male & $47(54)$ & $9(60)$ & $38(53)$ & \\
\hline Female & $40(46)$ & $6(40)$ & $34(47)$ & \\
\hline Race & & & & 1.0 \\
\hline White & 79 (91) & $15(100)$ & $64(89)$ & \\
\hline Black & $4(5)$ & $0(0)$ & $4(6)$ & \\
\hline Asian & $3(3)$ & $0(0)$ & $3(4)$ & \\
\hline Declined to answer & $1(1)$ & $0(0)$ & $1(1)$ & \\
\hline Ethnicity & & & & 1.0 \\
\hline Hispanic & $3(3)$ & $0(0)$ & $3(4)$ & \\
\hline Not Hispanic/Latino & $84(97)$ & $15(100)$ & $69(96)$ & \\
\hline RUCC grouping & & & & .52 \\
\hline Metro (RUCC 1-3) & $65(75)$ & $10(67)$ & $55(76)$ & \\
\hline Nonmetro (RUCC 4-9) & $22(25)$ & $5(33)$ & $17(24)$ & \\
\hline Insurance & & & & .34 \\
\hline Yes & $79(91)$ & $15(100)$ & $64(89)$ & \\
\hline No & $8(9)$ & $0(0)$ & $8(11)$ & \\
\hline Metastatic at diagnosis & & & & 1.0 \\
\hline Yes & $13(15)$ & $2(13)$ & $11(15)$ & \\
\hline No & $74(85)$ & $13(87)$ & $61(85)$ & \\
\hline Chemotherapy & & & & .37 \\
\hline Yes & $60(69)$ & $12(80)$ & $48(67)$ & \\
\hline No & $27(31)$ & $3(20)$ & $24(33)$ & \\
\hline Radiation therapy & & & & .78 \\
\hline Yes & $39(45)$ & $6(40)$ & $33(46)$ & \\
\hline No & $48(55)$ & $9(60)$ & $39(54)$ & \\
\hline Genetics discussion documented & & & & $<.001^{\mathrm{a}}$ \\
\hline Yes & $19(22)$ & $15(100)$ & $4(6)$ & \\
\hline No & $68(78)$ & $0(0)$ & 68 (94) & \\
\hline
\end{tabular}

Abbreviation: RUCC, Rural-Urban Continuum Codes.

${ }^{\text {a }}$ Statistically significant at $P<.05$.

follow-up by providers ( 1 was referred, but there were no contact notes from the genetics department), and reasons unknown $(\mathrm{n}=1)$. All 9 patients who received consultation (100\%) underwent genetic testing, 4 (44\%) of whom were identified to have a heritable cancer predisposition syndrome. Syndromes identified included NF1, hereditary paraganglioma-pheochromocytoma syndrome (PGL/PCC), FAP, and LFS (Table 2). Upon review of the types of genetic testing performed, 8 patients $(89 \%)$ had genotype testing and $1(11 \%)$ had karyotype testing to clarify that a translocation identified in her tumor was not germline.
Predictors of Genetic Consultation

Genetic referrals based on patient characteristics are detailed in Table 1. A statistically significant increased likelihood for genetic referral was seen in patients who had a documented genetics discussion with an oncology provider. There was no statistically significant difference in referrals to genetics based on age at diagnosis, sex, race, ethnicity, metropolitan versus nonmetropolitan residence, presence versus absence of insurance, localized versus metastatic cancer at time of diagnosis, and receipt of chemotherapy or radiation therapy. Notably, no patients who identified as Black, Asian, or Hispanic or who lacked 


\begin{tabular}{|c|c|c|c|c|}
\hline Total, $n$ & 87 & 15 & 72 & 4 \\
\hline Alveolar soft part sarcoma & $2(2.3)$ & $0(0)$ & $2(2.8)$ & NA \\
\hline Angiosarcoma & $3(3.4)$ & $1(6.7)$ & $2(2.8)$ & NA \\
\hline Chordoma & $1(1.1)$ & $0(0)$ & $1(1.4)$ & NA \\
\hline $\mathrm{CIC}$-rearranged sarcoma & $1(1.1)$ & $0(0)$ & $1(1.4)$ & NA \\
\hline Clear cell sarcoma & $1(1.1)$ & $0(0)$ & $1(1.4)$ & NA \\
\hline Dedifferentiated liposarcoma & $1(1.1)$ & $1(6.7)$ & $0(0)$ & NA \\
\hline Desmoid fibromatosis & $7(8.0)$ & $1(6.7)$ & $6(8.3)$ & 1 (25\%); FAP \\
\hline Fibrosarcoma & $1(1.1)$ & $0(0)$ & $1(1.4)$ & NA \\
\hline GIST & $2(2.3)$ & $2(13.3)$ & $0(0)$ & $2(50 \%) ; \mathrm{NF1}, \mathrm{PGL} / \mathrm{PCC}$ syndrome \\
\hline Giant cell tumor of bone & $2(2.3)$ & $0(0)$ & $2(2.8)$ & NA \\
\hline Inflammatory leiomyosarcoma & $1(1.1)$ & $0(0)$ & $1(1.4)$ & NA \\
\hline Kaposi sarcoma & $3(3.4)$ & $0(0)$ & $3(4.2)$ & NA \\
\hline Leiomyosarcoma & $4(4.6)$ & $1(6.7)$ & $3(4.2)$ & NA \\
\hline UPS & $1(1.1)$ & $0(0)$ & $1(1.4)$ & NA \\
\hline MPNST & $2(2.3)$ & $1(6.7)$ & $1(1.4)$ & NA \\
\hline Myxofibrosarcoma & $1(1.1)$ & $0(0)$ & $1(1.4)$ & NA \\
\hline Solitary fibrous tumor & $1(1.1)$ & $0(0)$ & $1(1.4)$ & NA \\
\hline Spindle cell sarcoma, NOS & $9(10.3)$ & $3(20.0)$ & $6(8.3)$ & NA \\
\hline Synovial sarcoma & $7(8.0)$ & $2(13.3)$ & $5(6.9)$ & NA \\
\hline
\end{tabular}

Abbreviations: DSRCT, desmoplastic small round cell tumor; FAP, familial adenomatous polyposis; GIST, gastrointestinal stromal tumor; LFS, Li-Fraumeni syndrome; MIFS, myxoinflammatory fibroblastic sarcoma; MPNST, malignant peripheral nerve sheath tumor; NA, not available; NF1, neurofibromatosis type 1; NOS, not otherwise specified; PGL/PCC, hereditary paraganglioma-pheochromocytoma; UPS, undifferentiated pleomorphic sarcoma.

health insurance were referred to genetics. Table 2 details genetics referrals by histologic subtype. Review of provider characteristics showed a trend toward increased genetics referrals in patients treated by the adult oncology team $(19 \% ; 14 / 73)$ versus pediatrics $(7 \% ; 1 / 14)$, with similar referrals from medical oncology $(18 \% ; 13 / 74)$ versus radiation oncology providers $(15 \% ; 2 / 13)$. In univariate analysis (Table 3), no associations were found between referral for genetic counseling and age at diagnosis, sex, metropolitan versus nonmetropolitan residential location, disease stage at diagnosis, and receipt of chemotherapy or radiation therapy.

\section{Discussion}

Genetic referral and testing are of critical importance for the identification of hereditary cancer predisposition syndromes, with implications for care, including surveillance, screening, and treatment. ${ }^{20-22}$ In all cancers, identification of such syndromes may inform therapy through identification of an actionable mutation, guide surveillance strategies, or allow application of cancer preventative measures, and it may influence family planning and support testing of at-risk family members. ${ }^{23,24}$ In sarcomas, such identification is particularly important, given implications for treatment in light of risk of therapy- 


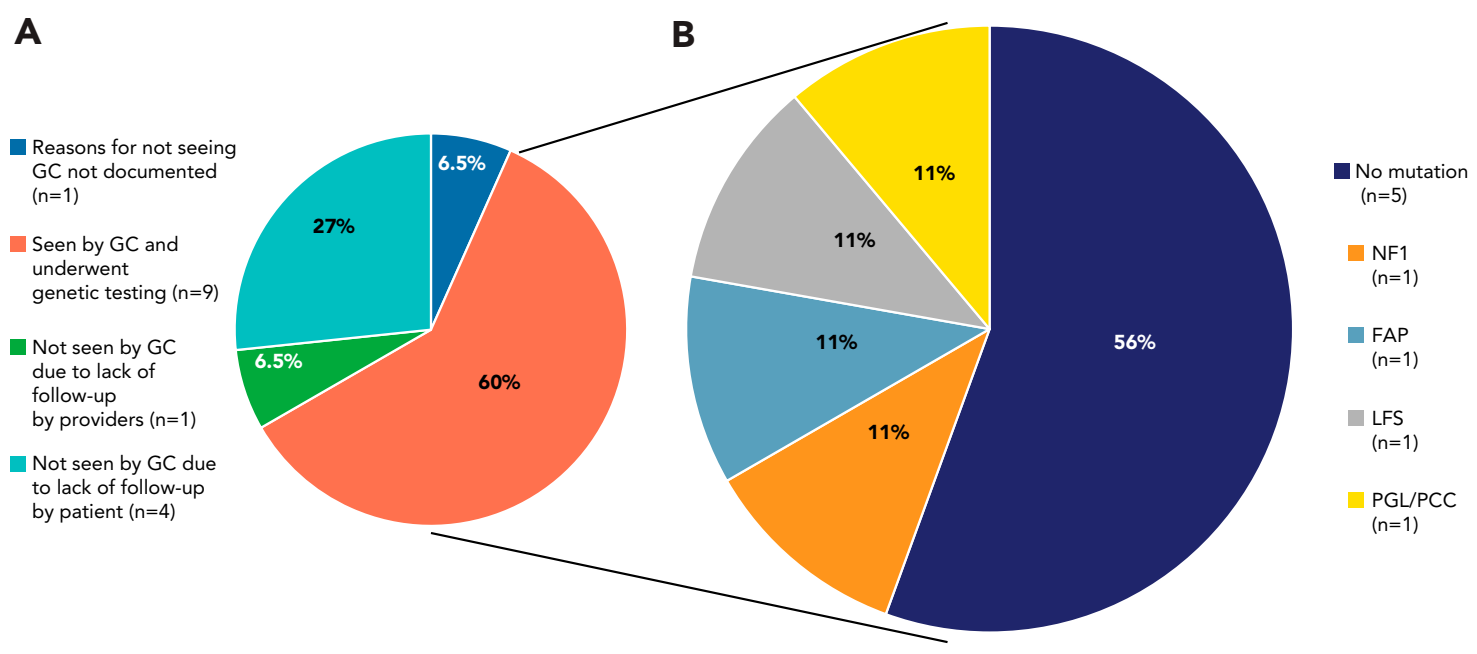

Figure 1. (A) Outcomes of referral for genetic counseling $(n=15)$ and $(B)$ results of genetic testing $(n=9)$.

Abbreviations: FAP, familial adenomatous polyposis; GC, genetic counselor; LFS, Li-Fraumeni syndrome; NF1, neurofibromatosis type 1; PGL/PCC, hereditary paraganglioma-pheochromocytoma syndrome.

related malignancies, such as consideration of radiation therapy and anthracycline-based chemotherapy for patients with LFS. $^{25}$ Despite a high frequency of pathogenic germline variants in sarcomas ${ }^{6,7,26}$ and in AYAs, ${ }^{5,26}$ we found that only $17 \%$ of AYA patients with sarcoma treated at an NCI-designated Cancer Center had genetic counseling referrals. Because this referral rate is even lower than previously published rates for genetic

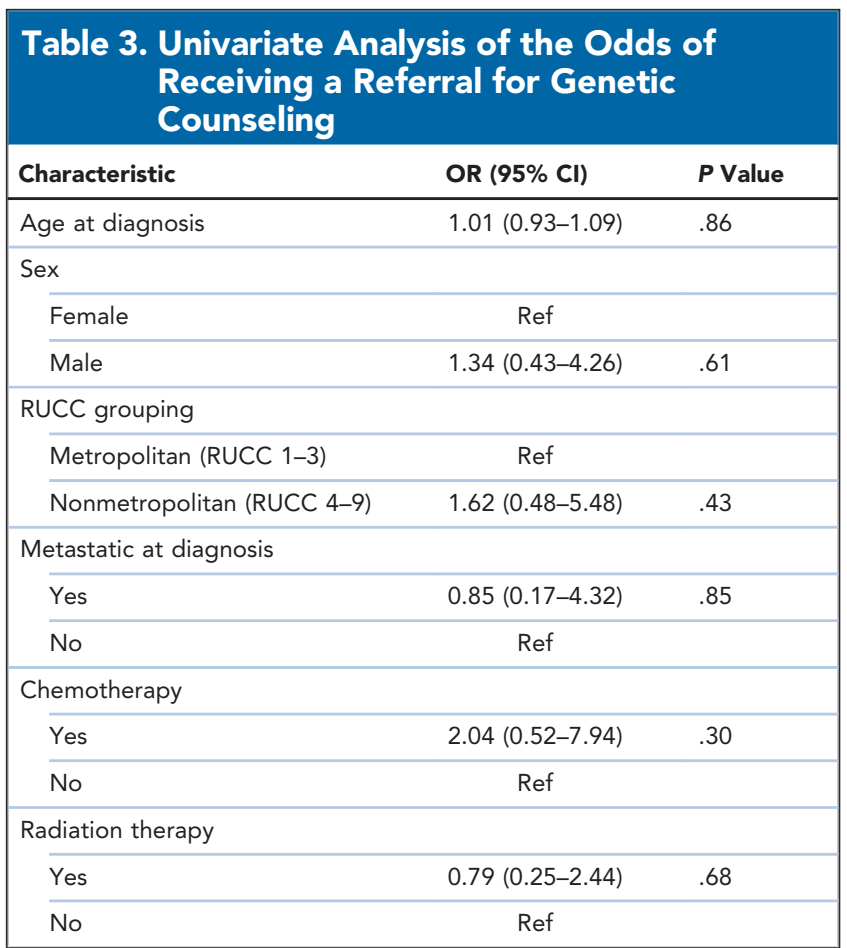

Abbreviations: OR, odds ratio; RUCC, Rural-Urban Continuum Codes. referrals ranging from $22 \%$ to $48 \%$ in other age groups and cancers, ${ }^{27-29}$ we sought to better understand factors influencing genetic consultations in AYA patients with sarcoma.

We found that referral for genetic consultation was linked most strongly to a documented genetics discussion with an oncology provider. Strikingly, despite NCCN Guidelines for AYA Oncology recommending documentation of a thorough family history and consideration for genetic consultation for all AYA patients with cancer, ${ }^{11}$ only $22 \%$ of the 87 AYA patients with sarcoma identified in our study had a documented discussion about genetics with an oncology provider. Other studies have similarly shown that failure of the treating oncologist to refer is the largest obstacle to receiving genetic counseling and/or testing. ${ }^{18,30}$ Although a variety of reasons for lack of genetics referral from the treating oncologist could be considered, including the absence of a high-risk personal or family history or concerns for insurance coverage, our study findings suggest that this is most often due to underconsideration of genetics by the oncology care team. In fact, only 1 patient in our study had documentation suggesting they were not referred for genetic consultation because the provider believed that the patient's personal and family history of cancer did not meet requirements for genetic counseling referral. Because genetic consultation is a component of the NCCN Guidelines for AYA Oncology and previous work has shown that higher compliance with NCCN Guidelines is associated with improved outcomes, ${ }^{31-35}$ the paucity of documentation of genetics discussions in the AYA patients in our study suggests a critical care need for these patients.

Although genetic referral is the first critical step in the process of genetic counseling and testing, there are several other possible areas of care breakdown, which our study 
also evaluated. Specifically, of the 15 patients referred to genetics, only $60 \%$ were seen in consultation, with the most common reason for referral without subsequent consultation being lack of follow-up by the patient. This may suggest possible reluctance for and barriers to genetic consultation among AYA patients. Previous studies have shown that AYAs at increased genetic risk have various challenges regarding the genetic counseling process, including the role of parents/partners/peers, health literacy, mental and emotional health, and available resources. ${ }^{36-40}$ Other work has shown that, given the potential of genetic testing decisions to affect recurrence and decisions about having children in the future, AYAs can be overwhelmed by genetic counseling considerations, ${ }^{41-43}$ which may result in the lack of patient follow-up seen in our study and may support ongoing educational initiatives regarding genetic consultation and testing in AYA patients. Workflow modifications should also be considered to avoid required previsit paperwork, which may be a barrier to care particularly for AYAs and was therefore discontinued at UW in 2019.

Despite a limited number of patients, all patients seen by genetics met testing criteria and underwent genetic testing. Supportive of the high frequency of hereditary predisposition cancer syndromes in AYA patients with sarcoma, $44 \%$ of patients who underwent genetic testing in our study had a heritable cancer predisposition syndrome identified, with 1 patient each with NF1, PGL/PCC, FAP, and LFS. Although our finding of genetic predisposition of $44 \%$ is in agreement with other findings, ${ }^{7,22,44}$ it is significantly higher than the documented $8 \%$ to $9 \%$ estimated frequency of germline mutations in cancer-predisposing genes in all AYA patients with cancer. ${ }^{5}$ Although it is an important finding, the small number of patients in our study limits interpretation of germline mutation frequency in this population. In addition, selection bias cannot be disregarded, because patients who have a more concerning phenotype of family history may be more likely to be referred or to receive follow-up with consultation and testing.

The high burden of genetic risk in AYA patients with sarcoma and the low rates of provider-documented genetics discussion and subsequent consultation highlight the need for more universal genetic counseling in AYA patients with sarcoma. Although family history is an important component of a comprehensive genetics assessment, we argue that oncology providers are not appropriately instructed on how to complete a comprehensive 3-generation pedigree, which is the gold standard family history for determining risk of hereditary genetic predisposition syndromes, ${ }^{29}$ and that such evaluation is labor-intensive and difficult to implement in a busy oncology practice. ${ }^{45}$ In addition, hereditary cancer syndromes associated with sarcomas can have a variety of presentations not always captured in a family history of cancer, such as de novo mutations (FAP and LFS), variable expressivity (NF1), or sex-dependent inheritance requiring professional genetic evaluation for appropriate testing options. Given evidence suggesting that family history of cancer may not capture more complex presentations of hereditary cancer syndromes, ${ }^{16,21,46}$ we suggest that a more universal recommendation should be made for genetic counseling for all AYA patients with sarcoma. Although such a broad recommendation would include a group of extraordinarily heterogeneous tumors with differences in driver aberrations and genetic complexity that affect the risk of hereditary cancer predisposition syndromes, our genetic understanding of sarcomas is limited because the focus has historically been on TP53 testing, with panel testing only more recently used. Even LFS as the most well-known hereditary predisposition syndrome in sarcomas continues to have refinements to clinical and genetic features, and there is emerging consideration of putative genetic causes for predisposition even in rare cases of Kaposi sarcoma, such as through inherited immunodeficiency syndromes. ${ }^{47}$ Although the syndromes identified in our study were observed in the sarcoma histologic subtypes more commonly associated with genetic predisposition syndromes, we believe universal genetic counseling for all sarcoma subtypes would serve to obtain a more accurate family history for syndrome consideration and further genetic research, establish care for future testing, and provide counseling benefits for this vulnerable population. We believe that such a universal recommendation for genetic counseling for all AYA patients with sarcoma is supported by our study findings and would help to improve genetic counseling use.

A notable limitation of our study includes the retrospective nature of the chart review. Although it is possible that documentation of discussions about genetics in the EHR may not accurately reflect whether providers considered or even engaged patients in a conversation about genetic consultation, we argue that best practice supports documentation of these discussions and that lack of documentation suggests no or limited consideration of genetics in these patients. We also declined to include documentation of family cancer history despite its potential impact on the decision to refer patients for genetic consultation, because documented family history data are frequently pulled from other parts of the EHR and may not represent meaningful documentation by the oncology provider. Small sample size may also limit the generalizability of this study; however, given the relative rarity of sarcoma diagnoses, the sample size in our study is not insignificant. Another important limitation with regard to the frequency of hereditary cancer predisposition syndromes identified in this study is that, although this study reviewed patients seen between 2015 and 2019, some genetic testing was 
performed before these dates and may have been restricted by available technology and insurance coverage of the time. The genetic tests performed were variable, ranging from single-site gene testing to multisystem panel testing, which limits our interpretation of results, especially related to negative test results. Finally, the low overall numbers of patients referred to genetics in our study may restrict the statistical analysis of variable association with referral to genetics. Considering this, although there was no statistically significant difference in referrals to genetics based on race, ethnicity, or presence or absence of insurance, this may be due to the relatively low numbers of patients referred to genetics. Notably, no patients in our study who identified as Black, Asian, or Hispanic or who lacked health insurance were referred to genetics. This consideration is critical because several studies have reported that minority patients' needs for genetic discussions and testing are more likely to go unmet ${ }^{48-50}$ whereas other studies have not observed different genetic counseling referral rates based on race and ethnicity. ${ }^{51,52}$ These incongruencies suggest the need for further research to explore the relationship between race, ethnicity, and socioeconomic disparities and genetic resources.

\section{Conclusions}

To our knowledge, this is the first study to report factors influencing genetic consultations in AYA patients with sarcoma. Despite the recommendation for consideration for genetic counseling in AYA patients, less than $25 \%$ of such patients in our study had a documented discussion about genetics. Almost all patients with such documentation were ultimately referred to genetics, suggesting this initial conversation by a provider is critical to genetics referral and should occur for all AYA patients with sarcoma. Supportive of the need for genetic consultations in these patients, all patients referred to genetics met criteria for genetic testing and $44 \%$ of patients who underwent genetic testing were found to have a heritable cancer predisposition syndrome. We believe that these data support consideration of a universal recommendation for genetic counseling in all AYA patients with sarcoma.

Submitted October 29, 2020; final revision received February 27, 2021; accepted for publication March 3, 2021.

Published online October 19, 2021.

Author contributions: Study design: McKay, Parkes. Data collection: McKay, Parkes. Data Interpretation: All authors. Writing-manuscript: All authors. Writing - review \& editing: All authors.

Disclosures: The authors have disclosed that they have not received any financial consideration from any person or organization to support the preparation, analysis, results, or discussion of this article.

Funding: This work was supported by the Shapiro Summer Research Program at the University of Wisconsin School of Medicine and Public Health.

Correspondence: Grace E. McKay, MPH, 2204 Chadbourne Avenue, Madison, Wisconsin 53726.

Email: geshea@wisc.edu

\section{References}

1. NCl Surveillance, Epidemiology, and End Results Program. Cancer stat facts: cancer among adolescents and young adults (AYAs) (ages 15-39). Accessed July 27, 2020. Available at: https://seer.cancer.gov/statfacts/ html/aya.html

2. Bleyer A, Viny A, Barr R. Cancer in 15- to 29-year-olds by primary site. Oncologist 2006;11:590-601.

3. Bleyer A, Barr R, Hayes-Lattin B, et al. The distinctive biology of cancer in adolescents and young adults. Nat Rev Cancer 2008; 8:288-298.

4. Fernebro J, Bladström A, Rydholm A, et al. Increased risk of malignancies in a population-based study of 818 soft-tissue sarcoma patients. Br J Cancer 2006;95:986-990.

5. Zhang J, Walsh MF, Wu G, et al. Germline mutations in predisposition genes in pediatric cancer. N Engl J Med 2015;373:2336-2346.

6. Mirabello L, Zhu B, Koster R, et al. Frequency of pathogenic germline variants in cancer-susceptibility genes in patients with osteosarcoma. JAMA Oncol 2020;6:724-734.

7. Ballinger ML, Goode DL, Ray-Coquard I, et al. Monogenic and polygenic determinants of sarcoma risk: an international genetic study. Lancet Oncol 2016;17:1261-1271

8. NIH, National Cancer Institute. Adolescents and young adults with cancer. Accessed July 23, 2020. Available at: https://www.cancer.gov/ types/aya

9. Mai PL, Best AF, Peters JA, et al. Risks of first and subsequent cancers among TP53 mutation carriers in the National Cancer Institute Li-Fraumeni syndrome cohort. Cancer 2016;122:3673-3681.

10. Daly MB, Pilarski R, Yurgelun MB, et al. NCCN Clinical Practice Guidelines in Genetic/Familial High-Risk Assessment: Breast, Ovarian, and Pancreatic. Version 1.2020. Accessed July 16, 2020. To view the most recent version, visit NCCN.org
11. Bhatia S, Pappo AS, Acquazzino M, et al. Adolescent and Young Adult (AYA) Oncology. Version 1.2020. Accessed May 25, 2020. To view the most recent version, visit NCCN.org

12. Li FP, Fraumeni JF Jr, Mulvihill JJ, et al. A cancer family syndrome in twentyfour kindreds. Cancer Res 1988;48:5358-5362.

13. Chompret A, Abel A, Stoppa-Lyonnet D, et al. Sensitivity and predictive value of criteria for p53 germline mutation screening. J Med Genet 2001; 38:43-47.

14. Bougeard G, Renaux-Petel M, Flaman JM, et al. Revisiting Li-Fraumeni syndrome from TP53 mutation carriers. J Clin Oncol 2015;33:2345-2352.

15. von Mehren M, Kane JM, Bui MM, et al. NCCN Clinical Practice Guidelines in Oncology: Soft Tissue Sarcoma. Version 2.2020. Accessed July 16, 2020. To view the most recent version, visit NCCN.org

16. Sussner KM, Jandorf L, Valdimarsdottir HB. Educational needs about cancer family history and genetic counseling for cancer risk among frontline healthcare clinicians in New York City. Genet Med 2011;13: 785-793.

17. Maradiegue A, Jasperson K, Edwards QT, et al. Scoping the family history: assessment of Lynch syndrome (hereditary nonpolyposis colorectal cancer) in primary care settings-a primer for nurse practitioners. J Am Acad Nurse Pract 2008;20:76-84.

18. Swink A, Nair A, Hoof $P$, et al. Barriers to the utilization of genetic testing and genetic counseling in patients with suspected hereditary breast and ovarian cancers. Proc Bayl Univ Med Cent 2019;32:340-344.

19. US Department of Agriculture. 2013 Rural-Urban Continuum Codes. Last updated October 25, 2019. Accessed July 1, 2020. Available at: https:// www.ers.usda.gov/data-products/rural-urban-continuum-codes

20. Farid M, Ngeow J. Sarcomas associated with genetic cancer predisposition syndromes: a review. Oncologist 2016;21:1002-1013. 
21. Mitchell G, Ballinger ML, Wong $S$, et al. High frequency of germline TP53 mutations in a prospective adult-onset sarcoma cohort. PLoS One 2013;8: e69026.

22. Toguchida J, Yamaguchi T, Dayton SH, et al. Prevalence and spectrum of germline mutations of the p53 gene among patients with sarcoma. N Engl J Med 1992;326:1301-1308.

23. McGee RB, Nichols KE. Introduction to cancer genetic susceptibility syndromes. Hematology (Am Soc Hematol Educ Program) 2016;2016:293301.

24. Rich TA, Liu M, Etzel CJ, et al. Comparison of attitudes regarding preimplantation genetic diagnosis among patients with hereditary cancer syndromes. Fam Cancer 2014;13:291-299.

25. Frebourg $T$, Bajalica Lagercrantz $S$, Oliveira $C$, et al. Guidelines for the Li-Fraumeni and heritable TP53-related cancer syndromes. Eur J Hum Genet 2020;28:1379-1386.

26. Sundar R, McVeigh T, Dolling D, et al. Clinical outcomes of adolescents and young adults with advanced solid tumours participating in phase I trials. Eur J Cancer 2018;101:55-61.

27. Febbraro T, Robison K, Wilbur JS, et al. Adherence patterns to National Comprehensive Cancer Network (NCCN) guidelines for referral to cancer genetic professionals. Gynecol Oncol 2015;138:109-114.

28. Meyer LA, Anderson ME, Lacour RA, et al. Evaluating women with ovarian cancer for BRCA1 and BRCA2 mutations: missed opportunities. Obstet Gynecol 2010;115:945-952.

29. Wood ME, Kadlubek P, Pham TH, et al. Quality of cancer family history and referral for genetic counseling and testing among oncology practices: a pilot test of quality measures as part of the American Society of Clinical Oncology Quality Oncology Practice Initiative. J Clin Oncol 2014;32:824829.

30. Shaw J, Bulsara C, Cohen PA, et al. Investigating barriers to genetic counseling and germline mutation testing in women with suspected hereditary breast and ovarian cancer syndrome and Lynch syndrome. Patient Educ Couns 2018;101:938-944.

31. Visser BC, Ma Y, Zak Y, et al. Failure to comply with NCCN guidelines for the management of pancreatic cancer compromises outcomes. HPB (Oxford) 2012;14:539-547.

32. Adegboyega TO, Landercasper J, Linebarger JH, et al. Institutional review of compliance with NCCN guidelines for breast cancer: lessons learned from real-time multidimensional synoptic reporting. J Natl Compr Canc Netw 2015;13:177-183.

33. Worhunsky DJ, Ma Y, Zak Y, et al. Compliance with gastric cancer guidelines is associated with improved outcomes. J Natl Compr Canc Netw 2015;13:319-325

34. Thiels CA, Hanson KT, Habermann EB, et al. Integrated cancer networks improve compliance with national guidelines and outcomes for resectable gastric cancer. Cancer 2020;126:1283-1294.

35. Lewis CM, Hessel AC, Roberts DB, et al. Prereferral head and neck cancer treatment: compliance with National Comprehensive Cancer Network treatment guidelines. Arch Otolaryngol Head Neck Surg 2010;136:12051211.
36. Codori AM, Zawacki KL, Petersen GM, et al. Genetic testing for hereditary colorectal cancer in children: long-term psychological effects. Am J Med Genet A 2003;116A:117-128.

37. Duncan RE, Young MA. Tricky teens: are they really tricky or do genetic health professionals simply require more training in adolescent health? Per Med 2013;10:589-600.

38. McConkie-Rosell A, Spiridigliozzi GA, Melvin E, et al. Living with genetic risk: effect on adolescent self-concept. Am J Med Genet C Semin Med Genet 2008;148C:56-69.

39. Michie S, Bobrow M, Marteau TM. Predictive genetic testing in children and adults: a study of emotional impact. J Med Genet 2001;38:519-526.

40. Pichini A, Shuman C, Sappleton K, et al. Experience with genetic counseling: the adolescent perspective. J Genet Couns 2016;25:583-595.

41. Sawyer SM, McNeil R, McCarthy M, et al. Unmet need for healthcare services in adolescents and young adults with cancer and their parent carers. Support Care Cancer 2017;25:2229-2239.

42. McCarthy MC, McNeil R, Drew S, et al. Information needs of adolescent and young adult cancer patients and their parent-carers. Support Care Cancer 2018;26:1655-1664.

43. Lake PW, Kasting ML, Dean M, et al. Exploring patient and provider perspectives on the intersection between fertility, genetics, and family building. Support Care Cancer 2020;28:4833-4845.

44. Ognjanovic S, Olivier M, Bergemann TL, et al. Sarcomas in TP53 germline mutation carriers: a review of the IARC TP53 database. Cancer 2012;118: 1387-1396.

45. Rich EC, Burke W, Heaton CJ, et al. Reconsidering the family history in primary care. J Gen Intern Med 2004;19:273-280.

46. Wakefield CE, Quinn VF, Fardell JE, et al. Family history-taking practices and genetic confidence in primary and tertiary care providers for childhood cancer survivors. Pediatr Blood Cancer 2018;65:e26923.

47. Byun M, Ma CS, Akçay A, et al. Inherited human OX40 deficiency underlying classic Kaposi sarcoma of childhood. J Exp Med 2013;210:17431759.

48. Jagsi R, Griffith KA, Kurian AW, et al. Concerns about cancer risk and experiences with genetic testing in a diverse population of patients with breast cancer. J Clin Oncol 2015;33:1584-1591.

49. Muller C, Lee SM, Barge W, et al. Low referral rate for genetic testing in racially and ethnically diverse patients despite universal colorectal cancer screening. Clin Gastroenterol Hepatol 2018;16:1911-1918.e2.

50. Manrriquez E, Chapman JS, Mak J, et al. Disparities in genetics assessment for women with ovarian cancer: can we do better? Gynecol Oncol 2018; 149:84-88.

51. Barcenas $\mathrm{CH}$, Shafaee $M N$, Sinha $A K$, et al. Genetic counseling referra rates in long-term survivors of triple-negative breast cancer. J Natl Compr Canc Netw 2018;16:518-524.

52. Stuckey A, Febbraro T, Laprise J, et al. Adherence patterns to National Comprehensive Cancer Network guidelines for referral of women with breast cancer to genetics professionals. Am J Clin Oncol 2016;39: 363-367. 